\title{
$+$ \\ el cemento blanco y el cemento coloreado en la construcción de carreteras
}

\section{MEYER}

*Journal de la Construetion de la Suisse Romande» $N^{:}$1, 15 Enexo 1962, pag. 31 y N.* 2, 31 Enero 1962, pág. 34 *

El cemento blanco danés es un cemento Portland fabricado por medio de materias primas muy puras y pobres en hierro; se clinkeriza en hornos rotatorios calentados con mazout, lo que implica que incluso los clínkeres sean blancos.

La blancura crece durante la molienda y en función de Ia flnura, pero este cemento, a pesar de todo, no se muele más fino que el cemento portland de endurecimiento rápido de tipo corriente. Cumple las normas suizas de cemento Portland de endurecimiento rápido y alta resistencia. Su retracción es menos importante que la de otros cementos de endurecimiento rápido.

Si algunos creen que el cemento blanco tiene mayor retracción y da más fisuras capilares, es porque se tiene tendencia a emplear mezclas muy húmedas y grasas.

Su resistencia crece rápidamente, porque su endurecimiento es también rápido. Su relación aguacemento-resistencia se calcula según la fórmula de Bolomey.

Los cementos coloreados se fabrican, o bien a base de cemento blanco para todos los colores delicados y claros, o bien a base de cemento gris para los colores oscuros.

El cemento coloreado se compone, pues, de cemento Portland mezclado con materias colorantes en el curso de la molienda; no es, por tanto, un cemento portland según la definición habitual de éste. El colorante se añade normalmente en la proporción del $5 \%$ con relación al peso del cemento $\mathrm{y}$, en casos especiales, del $10 \%$ como máximo.

En consecuencia, si se considera la materia colorante como una parte del agregado del hormigón coloreado, para una relación $1: 5$ no supone más que el $1 \%$ y a lo más el $2 \%$ del cemento, lo cual es insignificante. Por ello, los Pliegos de Condiciones daneses para hormigón permiten emplear, sin más, los cementos coloreados para la fabricación del hormigón ordinario y lo mismo del hormigón armado, a condición de que la materia colorante no favorezca la oxidación de las armaduras de hierro, lo cual no es el caso de las materias colorantes empleadas por nosotros.

Los colores están sometidos a severas exigencias, ya qute, aun siendo inalterables a la luz y resistentes a la intemperie, deben poder resistir a la acción de los álcalis y de la cal que se forman en el hormigón durante su endurecimiento. Para los amarillos, pardorrojizos o negros se emplea, generalmente, óxido de hierro, y, para el verde, oxido de cromo. Son colores inorganicos, pues hay pocos orgánicos utilizables. Esto limita, evidentemente, el número de colores con los que es posible producir los cementos coloreados, pero con estos colorantes se cubre ya una buena parte de la gama de colores.

Al fabricar un hormigón blanco a coloreado, no sólo el cemento influye en el aspecto del hormigón, sino también los agregados. Para el homigón blanco se deben utilizar agregados blancos, lo que da, evidentemente, el color más claro y más bello. Sin embargo, el empleo del cemento blanco junto con agregados de tipo corriente - sobre todo cloros-puede dar, iguaimente, un resultado relativamente satisfactorio. Cuando se trata de cemento blanco para la construcción de carreteras, es preciso ser exigente, no sólo para el color de los agregados, sino también para la resistencia al desgaste. En este punto, nos encontramos en presencia de una limitación destacada. Existen variós tipos de agregados blancos. La mayor parte de los que se encuentran en la naturaleza son mármoles, que son poco resistentes al desgaste. Los vehiculos no marchan más que excepcionalmente al borde de la pista y, entonces, el empleo de mármol como agregado del bordilio de la calzada en hormigón blanco queda justificado. Si es preciso un pulido o usar el hormigón con fines distintos a la construcción de carreteras, es evidente que el mármol presenta la gran ventaja de reducir el trabajo de pulido. 
Hay dos agregados particularmente sólidos y resistentes al desgaste. Ninguno de ellos se encuentra en la naturaleza: uno es semiartificial-sílex calcinado-y el otro se prepara en fábrica-synopal-.

El sílex calcinado es producido por una recocción en el curso de la cual se transforma: su color sombra pasa a blanco, pero a la vez sufre una fisuración en cierta medida. Estas fisuras no tienen influencia en la resistencia al hielo del silex calcinado, de tal suerte que no hay que temer en el empleo del silex calcinado para una banda de calzada de hormigón, pues es un material muy resistente al desgaste.

El «synopal» se obtiene con la misma materia prima que el vidrio poco más o menos, pero así como en la fabricación de éste se evita, en lo posible, la formación de cristales, en la fabricación del usynopal» se provoca, por el contrario, una microcristalización. Es un producto de alta resistencia que da un hormigón resistente al hielo y al desgaste y que es, a la vez, antideslizante. Este material refleja la luz de los faros de los vehículos.

Para el hormigón coloreado se deben elegir los agregados teniendo en cuenta el color deseado. Podrá ser cuestión de mármol verde, diabasa y pórfido en sus diversos colores, pero este problema depende de los materiales pétreos que se encuentran en los diferentes países.

¿Cuáles son las propiedades exigidas a un hormigón que ha de utilizarse, por ejemplo, para la construcción de la banda blanca que nos interesa a nosotros? Este hormigón debe presentar, ante todo, una gran resistencia mecánica, ser suficientemente resistente al desgaste y, como ya queda dicho, antideslizante.

No es necesario demostrar que estas propiedades pueden alcanzarse y, de hecho, deben conseguirse. Se puede llegar con un hormigón blanco e, incluso, coloreado, pero es importante que el hormigón esté fabricado correctamente, de tal suerte que la obtención de las buenas propiedades buscadas no lleve consigo inconvenientes tales como una gran retracción, una fisuración, etc. Estos defectos deben ser evitados en la medida de lo posible y pueden serlo mediante una fabricación adecuada y, sobre todo, por un tratamiento ulterior correcto.

¿Qué es, pues, un hormigón hecho con cemento blanco o cemento coloreado?

Es de señalar, en primer lugar, que los agregados se eligen a menudo, desgraciadamente, teniendo en cuenta su precio y despreciando su aspecto. Debe, naturalmente, tenerse en cuenta la facilidad de adquisición de dichos materiales.

A este propósito, conviene señalar que son los elementos finos-diámetros comprendidos entre 4 y 8 milímetros-los que tienen una influencia decisiva sobre el color, incluso aunque los elementos más gruesos influyan, en cierta medida, sobre el carácter del hormigón.

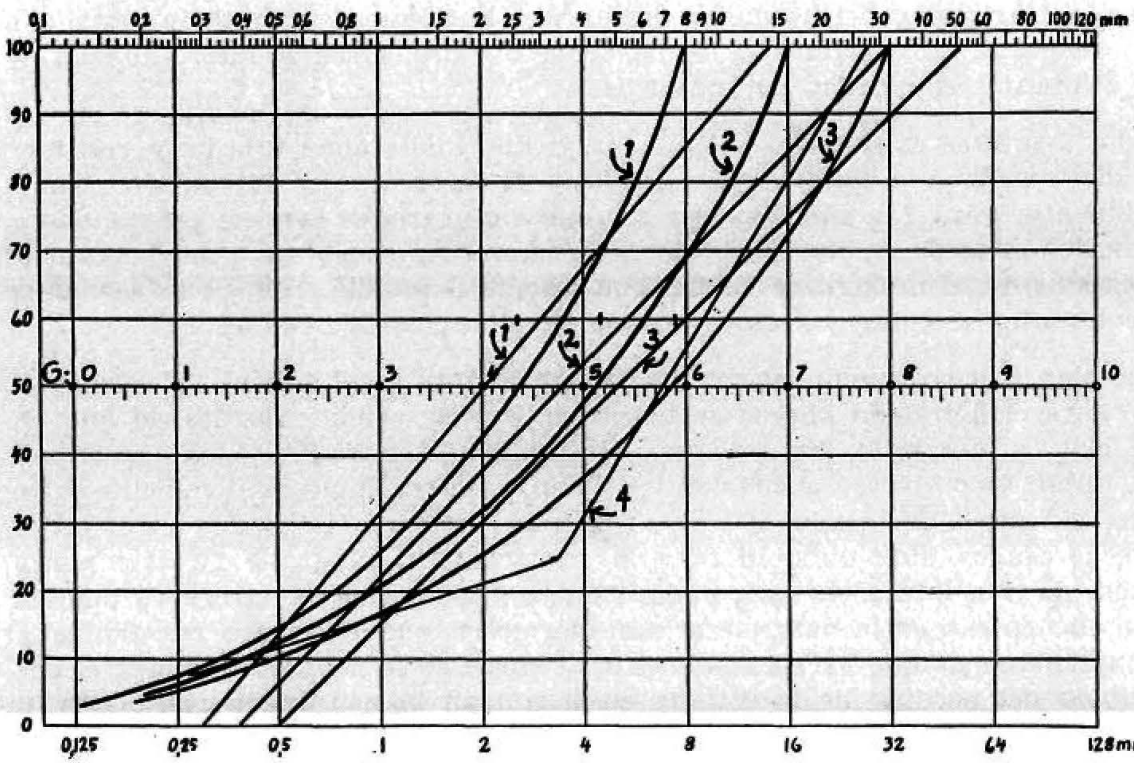

Fig. 1. Curvas granulométricas continuas para agregados de hormigón. La ourva 2 para una capa de hormigón de $5 \mathrm{~cm}$ de espesor; la curva 3 para una capa de hormigón de 10 a $20 \mathrm{~cm}$ de espesor. Si la capa de hormigón blanco de 5 em de espebase de plica sobre una losá mumentarso no podra aumentarse. La de $8 \mathrm{~mm}$ de $\phi$ puede dar un hormigón que facilite la puesta en obra. 
Fig. 2. Relación entre la resistencia a la compremigón flúido.

Se olvida con frecuencia el comprobar si la curva granulométrica de los materiales es correcta, la cual condiciona una fabricación satisfactoria de un hormigón de buena calidad. Evidentemente, es posible producir un hormigón con áridos de calidad inferior a los mencionados, pero siempre es preciso esforzarse en Lograr una buena calidad, es decir, de acuerdo con una curva granulométrica adecuada. En la figura 1 se indican más o menos las curvas granulométricas que aseguran una calidad satisfactoria. sión y el consumo de cemento: Curva 1: Hormigón seco,-Curva 2: Hormigón plástico,-Curva 3: Hor-

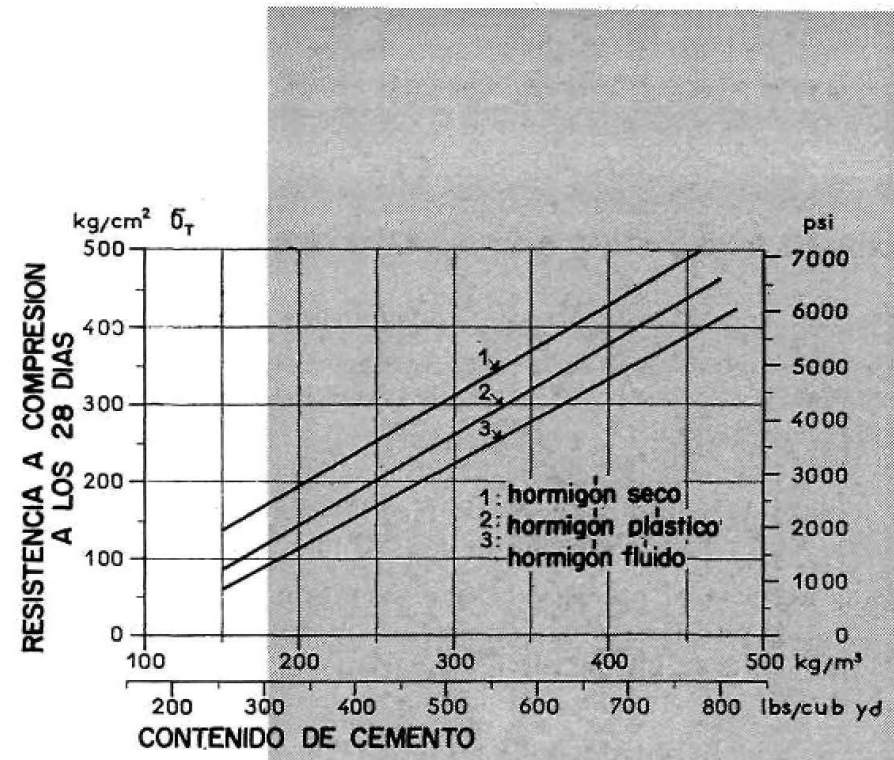

Representamos la curva granulométrica en un diagrama de tamizado idéntido al empleado en los países escandinavos, partiendo de $1 / 8 \mathrm{de} \mathrm{mm}$ y doblando sucesivamente hasta lograr el máximo; en otras palabras, tenemos un sistema de tamizado simple, que présenta ciertas ventajas. Calculamos el módulo de finura como la superficie de la parte que, sobre el diagrama, se encuentra por encima de la curva granulométrica, tomando como unidad la superficie situada entre dos abscisas consecutivas.

Sobre la ordenada $50 \%$, colocamos en abscisas los puntos $0-1-2-3$, etc., partiendo de $1 / 8 \mathrm{de} \mathrm{mm}$. Trazamos ahora una línea oblicua que determine una superficie igual a la delimitada por la curva granulométrica. La intersección de esta línea oblicua con la horizontal en la ordenada $50 \%$ da el módulo de finura. Así observamos en el material fino un módulo de finura de 4, y en el material grosero un módulo de finura de 5,4 .

Es muy raro encontrar en la naturaleza materiales que presenten curvas granulométricas que correspondan a la indicada y es preciso preparar mezclas con curvas granulométricas correctas mezclando elementos finos y gruesos. Los elementos cuyo tamaño máximo de grano es de $8 \mathrm{~mm}$ se destinan, por ejemplo, al hormigón coloreado que se ha de pulir (piedra artificial), donde los granos deben ser finos mientras que la curva del centro va mejor al hormigón del que estamos tratando.

La fase siguiente de la fabricación del hormigón es determinar la cantidad de agua necesaria. Este es el punto de partida en la determinación de las proporciones de mezcla. No se puede añadir cemento y decir que es necesario emplear una proporción de $1: 6$ ó $1: 5$, pues esto sería irracional. Lo que se debe hacer es fijar la composición real del hormigón y determinar el consumo de agua, porque se sabe que todo árido exige una cantidad determinada de agua para un hormigón de cierta consistencia. Esta cantidad de agua no depende, por así decir, del hecho de que se trate de un hormigón graso o magro. Es la ley denominada de Lyse, presentada por el ingeniero noruego Inge Lyse, profesor en Trondheim.

Lo siguiente es, naturalmente, saber cuál es la cantidad de agua necesaria, una vez conocidos el módulo de finura y el asentamiento.

Fig. 3. Comparación entre el contenido de aire y las resistencias de los diferentes hormigones.

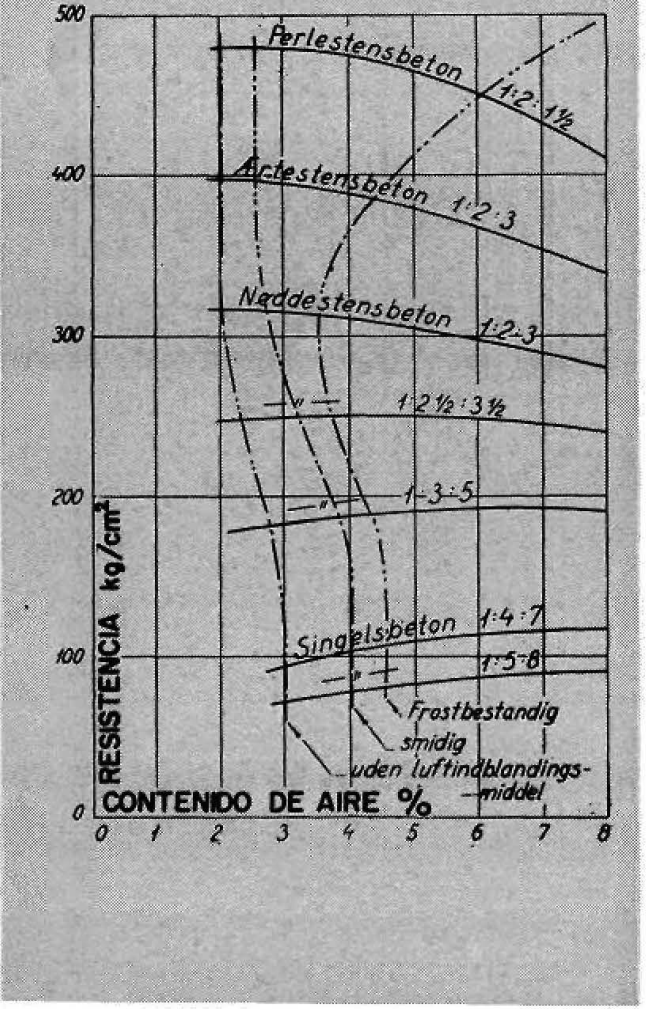


El autor presenta una proposición para determinar el consumo de agua, hecha por el ingeniero Glarbo, de la Escuela de Altos Estudios de Dinamarca, quien ha indicado la siguiente fórmula:

$$
\text { Consumo de agua }=k \times \frac{700+9 \times f m^{2}+13 s}{f m},
$$

ecuación en la que $f m$ es el módulo de finura, $s$ el asentamiento, y $k$ una constante de la que volveremos a hablar.

Si trazamos la curva granulométrica del árido elegido, es fácil determinar el módulo de finura y vigilar si la finura del árido se modiffca. Conviene, naturalmente, componer el agregado de al menos dos partes para mantenerlo constante.

Entonces es preciso elegir el asentamiento, que debe ser lo más bajo que permita la docilidad del hormigón destinado a la construcción de carreteras, entre 0 y $1 \mathrm{~cm}$, lo que es un hormigón espeso. En la figura 2 se puede ver un diagrama que representa, a grandes rasgos, la relación entre la resistencia a la compresión y la cantidad de cemento.

Con el hormigón seco se obtiene una mayor resistencia a la compresión, a igualdad de cantidad de cemento, y se alcanza una resistencia de 0,35 a $0,80 \mathrm{~kg} / \mathrm{cm}^{2}$ por cada kilo de cemento contenido en el hormigón flúido, contra 0,9 a $1,1 \mathrm{~kg} / \mathrm{cm}^{2}$ por kilo de cemento en un hormigón seco. En otras palabras, en el hormigón flúido se precisan de 3 a $1,25 \mathrm{~kg}$ de cemento por kilo de resistencia, y en el seco se necesitan de 1 a $0,9 \mathrm{~kg}$. La diferencia es tanto más notoria cuando la resistencia del hormigón es más débil.

Antes de fijar la composición del hormigón es preciso elegir la resistencia, a menos que se prefiera simplemente-al tratarse de un hormigón para carreteras-fijar una relación agua-cemento máxima, por ejemplo, de 0,5. La resistencia del hormigón blanco preparado con áridos resistentes de buena calidad puede valorarse en (cubos de $20 \mathrm{~cm}$ ):

$$
\sigma_{2 \&}=k\left(\frac{1}{a / c}-0,5\right)
$$

en la que $k=$ de 250 a $300 \mathrm{~kg} / \mathrm{cm}^{2}$ según el grado de frescura del cemento. Si ahora exigimos una resistencia de $300 \mathrm{~kg} / \mathrm{cm}^{2}$, nos encontramos con $k=250 \mathrm{~kg} / \mathrm{cm}^{2}$ :

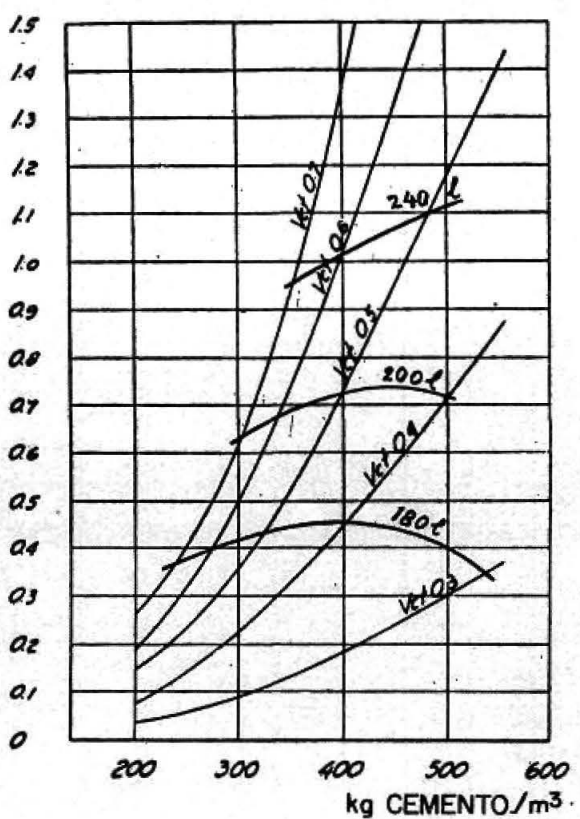

Fig. 4. Relación de retracción: agua-cemento : cemento por $\mathrm{m}^{3}$. Obsérvese, por ejemplo, la curva para 180 litros de agua, en la que la retracción decrece de 400 a 500 kilos por $\mathrm{m}^{3}$. Fig. 5. Los valores de retraceíon de la figura 4, or-
denados por el contenido de agua total por $\mathrm{m}^{3}$ ( $V$ kg) de hormigón.

$$
\begin{aligned}
& \frac{1}{a / c}-0,5=\frac{300}{250}=1,2 \\
& \frac{1}{a / c}=1,7 \quad ; \quad a / c=0,6
\end{aligned}
$$

Con $k=300 \mathrm{~kg} / \mathrm{cm}^{2}$, se obtiene $\frac{1}{a / c}=1,5 ; a / c=0,67$.

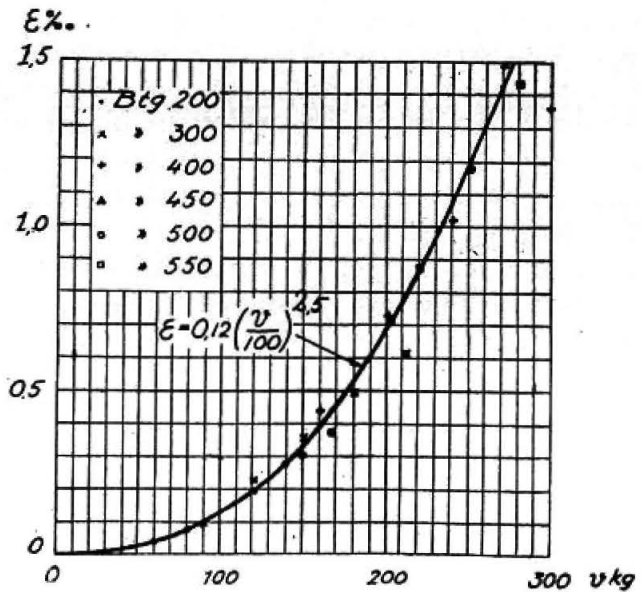



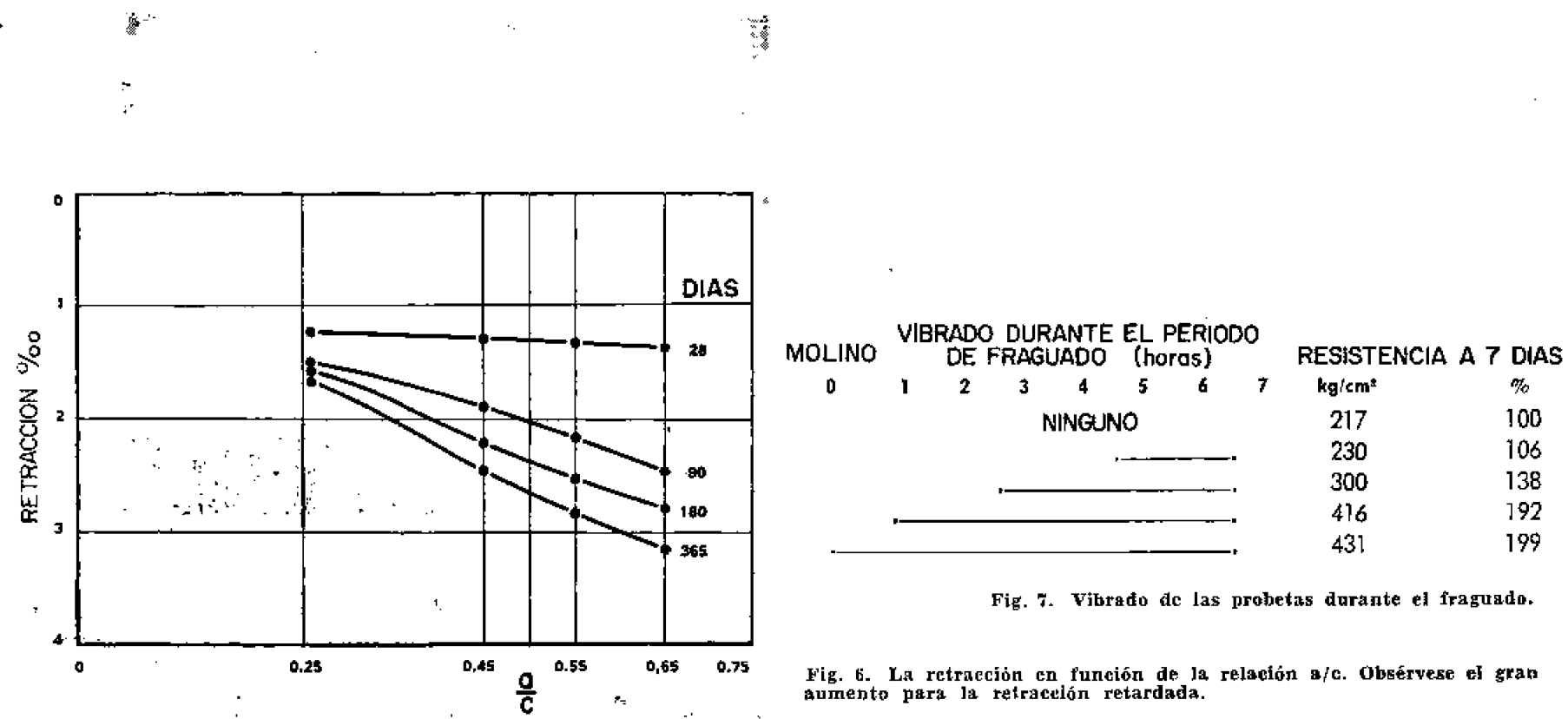

Fig. 7. Vibrado ac las probetas durante el fraguado.

E'ig. B. La retracción en función de la relación a/c. Obsérvese el gran aumento para la réractión retardada.

$\mathrm{Si}$, en consecuencia, nosotros elegimos la relación $a / c=0,5$, estamos seguros de tener ux buen margen de resistencia.

Si es preciso realizar una capa más espesa, debemos elegli $32 \mathrm{~mm}$ como tamaño máxímo de árído; pero si se trata sólo de $5 \mathrm{~cm}$ de hormigón blanco, habrá que considerar sólo de $16 \mathrm{~mm}$. Tendremos, entonces, un módulo de flnura de $4,8 \mathrm{y}$, en consecuencia, un consumo de agua de:

$$
\mathrm{v}=k \frac{700+9 \times 4,8^{2}+13 \times 1}{4,8}=k \times 192
$$

donde $\dot{k}$ varía de 0,85 a 1,1 , según que la grava sea redondeada 0 angulosa.

Como el sílex calcinado y el ksynopal son de aristas muy vivas, debemos considerar $k=1,1$, y entonces:

$$
\mathrm{V}=211 \text { litros }
$$

Finalmente, es preciso decidir si se quiere emplear un agente aireante y cuánto alre se requiere en el hormigon. En tanto nos movamos hasta $2 \%$ de aire, aproximadamente, no es preciso tenerlo en cuenta en el cálculo de resistencias, pero un exceso debe tomarse en consideración.

Si suponemos que elegimos $5 \%$ de aire, entonces tendremos una cantidad suplementaria de 30 litros por metro cúbico que dtsminuye la resistencia en la misma medida que el agua, Afortunadamente, se obtiene, a la vez, la posibilidad de reducir la cantidad de agua, por lo menos, en 10 litros $_{r}$ restando 200 , según el cálculo anterior.

Con una relación agua-cemento $=0,5$, debemos emplear $400 \mathrm{~kg}$ de cemento por $\mathrm{m}^{3}$, y obtenemos una relación agua-aire-cemento efectiva de

$$
\frac{230}{400}=0,58
$$

de tal manera que conservamos siempre la resistencia de $300 \mathrm{~kg} / \mathrm{cm}^{3}$.

\begin{tabular}{|c|c|c|}
\hline 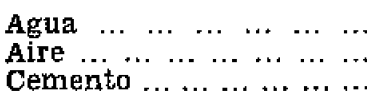 & $\begin{aligned} 200 \mathrm{~kg} \\
0 \mathrm{~kg} \\
400 \mathrm{~kg} \\
\end{aligned}$ & $\begin{array}{r}200 \text { litros } \\
50 \text { litros } \\
128 \text { litros }\end{array}$ \\
\hline Aridos & $\begin{array}{r}600 \mathrm{~kg} \\
1.648 \mathrm{~kg}\end{array}$ & $\begin{array}{l}378 \text { litros } \\
622 \text { litros }\end{array}$ \\
\hline & $2.248 \mathrm{~kg}$ & 00 \\
\hline
\end{tabular}

La composición propiamente dicha del hormigón se presenta, en este caso, como sigue, con un peso especiflco del cemento tal que $100 \mathrm{~kg}=32$ litros y para el árido de 2,65 :

La proporción de mezcla, en peso, es de $1: 4,12$.

Es preciso, naturalmente, realizar una mezcla de ensayo para llegar a la proporción de mezcla finaI por correcciones sucesivas. 
Si $400 \mathrm{~kg}$ de cemento por $\mathrm{m}^{*}$ no parece ser la proporción adecuada, podemos llevar la relación aguacemento a 0,55 , teniendo asi:

\begin{tabular}{|c|c|c|}
\hline $\begin{array}{llllllll}\text { Agua } & \ldots & \ldots & \ldots & \ldots & \ldots & \ldots \\
\text { Aire } & \ldots & \ldots & \ldots & \ldots & \ldots & \ldots & \ldots \\
\text { Cemento } & \ldots & \ldots & \ldots & \ldots & \ldots & \ldots\end{array}$ & $\begin{array}{r}200 \mathrm{~kg} \\
0 \mathrm{~kg} \\
364 \mathrm{~kg} \\
\end{array}$ & $\begin{array}{r}200 \text { litros } \\
50 \text { litros } \\
116 \text { litros } \\
\end{array}$ \\
\hline Aridos & $\begin{array}{r}564 \mathrm{~kg} \\
1.680 \mathrm{~kg}\end{array}$ & $\begin{array}{l}366 \text { litros } \\
634 \text { litros }\end{array}$ \\
\hline
\end{tabular}

donde resulta una proporción de mezcla en peso de 1:4,62.

Si empleamos un agente plastificante para reducir la cantidad de agua y que no consideramos más que sobre $2 \%$ de aire, tendremos una cantidad suplementarla de agregados de 30 litros.

Sin embargo, a juicio del autor, no hay duda de que el hormigón de $400 \mathrm{~kg}$ de cemento por $\mathrm{m}^{3}$ es el más indicado.

Muchos pensarán quizás que el hormigón con $400 \mathrm{~kg}$ de cemento tiene mayor retracción que el de $364 \mathrm{~kg}$, pero esto no es muy exacto. Es cierto que la retracción aumenta con la cantidad de cemento de la mezcla, o sea, el volumen absoluto de cemento g agua, pero también con la porasidad del mortero, lo que condiciona el grado de su higroscopia. El consumo de agua es el factor predominante en la reracción, lo que nos concuce a lo curva granulométrica y al módulo de finura.

La cantidad de cemento no disminuye más que de 328 a 316 litros por $\mathrm{m}^{3}$, es decir, de 3 a $4 \%$, mientras que la porosidad restante aumenta $6 \%$, lo que constituye la retracción de la pasta de cemento; en el propio hormigón será tan débil que quedará sin tener importancia, supuesto que el hormigón se realice $\mathrm{y}$ trate posterformente de una manera juiciosa.

Según Henk, «la opinión expresada en otro tiempo, y a menudo en nuestros dias, sobre la importancia decisiva de la retracción del cemento y de las exigencias que ello lleva consigo en cuanto a aglomerantes con un mínimo de retracción y molidos lo más groseramente posible (en consecuencia, de endurecimiento lento) o, incluso, cementos expansivus para el hormigón de construcción, no está justificado en modo alguno. Se puede cast siempre comprobar que los malos resultados no pueden atribuirse a los aglomerantes, sino a los defectos maniflestos en la fabricación y puesta en obra».

La mezcla propiamente dicha del hormigón debe efectuarse de una manera muy eficaz, ya que una mezcla conveniente permite reducir el consumo de agua. La compactación debe ser también muy eficaz; y si se vierte el hormigón blanco directamente sobre el gris fresco, no se corre el riesgo de que las capas se separen.

El autor ha hecho algunos ensayos que consistian en batir el hormigón una vez por minuto en diferentes momentos del periodo de fraguado. Esto significa que el hormigón no fue sometido a ningún otro desplazamlento que el provocado por el vibrado. Cuanto más tarde durante el periodo de fraguado se vibraba el hormigón, más aumentaba la resistencia, hasta $100 \%$, aproximadamente. Una vibración posterior eficaz da resultados análogos, pero con un aumento en la resistencia, más débil. Esto puede dar ciertas ideas en cuanto a la ejecución de la banda blanca. Este tratamiento posterior na dado también un hormigón de menor tendencla a la fisuración, tendencia medida según el método de anillo de Continho.

Cubos de hormigón: Dosifleación 1:6,2,

Relación agua-cemento: 0,6 (relación cemento-agua $=1,67$ ).

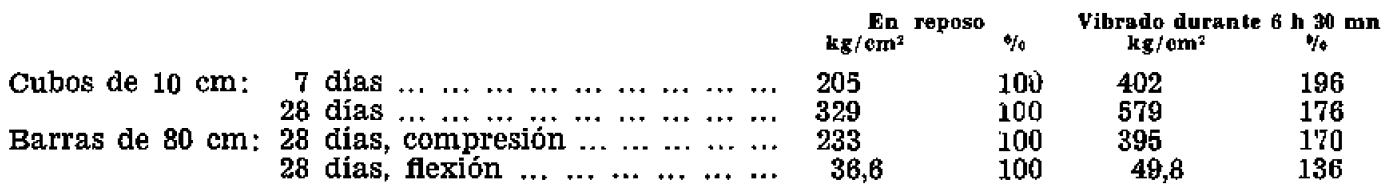

En lo que concierne a la ejecución propiamente dicha, el autor destaca la importancia en mantener el hormigón húmedo, preferentemente durante quince dias, $\mathrm{y}$, principalmente, antes de que comience la desecación, por ligera que sea, de la superficie. Tal desecación, incluso ligera, es muy peligrosa. sobre todo si es causada por el viento. Fisuras invisibles pueden formarse durante un riego posterior, aunque aparecen después de la desecación. En consecuencia, es preciso preservar el hormigón del sol y del viento y protegerlo lo más posible por medio de esteras, chapas o abrigos movibles. El empleo de arena puede ser peligroso en caso de helada y ptiede provocar desconchamientos del hormigón fresco. El scurado de membranas (tratamiento ulterior con una capa delgada de barniz impermeable) se considera por muchos como un buen método, pero su effcacia no puede compararse con la de un buen riego. 